\title{
Characterization and pathogenesis of anemia in glycogen storage disease type la and Ib
}

\author{
David Q. Wang, MD'1, Caroline T. Carreras'1, Laurie M. Fiske, BS1', Stephanie Austin, MS², \\ Danielle Boree, MS'3 , Priya S. Kishnani, MD² and David A. Weinstein, MD, MMSc ${ }^{1}$
}

Purpose: The aim of this study was to characterize the frequency and causes of anemia in glycogen storage disease type I.

Methods: Hematologic data and iron studies were available from 202 subjects (163 with glycogen storage disease Ia and 39 with glycogen storage disease Ib). Anemia was defined as hemoglobin concentrations less than the 5 th percentile for age and gender; severe anemia was defined as presence of a hemoglobin $<10 \mathrm{~g} / \mathrm{dl}$.

Results: In glycogen storage disease Ia, 68/163 patients were anemic at their last follow-up. Preadolescent patients tended to have milder anemia secondary to iron deficiency, but anemia of chronic disease predominated in adults. Severe anemia was present in 8/163 patients, of whom $75 \%$ had hepatic adenomas. The anemia improved or resolved in all 10 subjects who underwent resection of liver lesions. Anemia was present in $72 \%$ of patients with glycogen storage disease $\mathrm{Ib}$, and severe anemia occurred in $16 / 39$ patients. Anemia in patients

\section{INTRODUCTION}

The glycogen storage diseases (GSDs) are a group of disorders that are characterized by aberrant storage or utilization of glycogen. GSD type I (GSD I) is due to impaired glucose6-phosphatase activity, and it is the most severe of the hepatic glycogenoses from a hypoglycemia vantage point because this critical enzyme catalyzes the terminal step of both glycogenolysis and gluconeogenesis. As a result, endogenous glucose production is impaired or absent, and shunting of glucose-6phosphate into alternative pathways results in hyperlactatemia, hypertriglyceridemia, and hyperuricemia. ${ }^{1}$

Since the discovery of continuous glucose feeding in 1971 and uncooked cornstarch in 1982 as methods to maintain euglycemia, patient survival has improved greatly in the GSD I population. ${ }^{2}$ Long-term complications continue to occur, however, including hepatocellular adenoma formation with risk for carcinoma, chronic hepatic inflammation, gout, osteoporosis, focal segmental glomerulosclerosis, nephrocalcinosis, and anemia. ${ }^{3}$ In addition, patients with GSD Ib, who have defects in the glucose-6-phosphate transporter, have additional manifestations, including neutropenia, neutrophil dysfunction, and inflammatory bowel disease. ${ }^{4}$ with glycogen storage disease Ib was associated with exacerbations of glycogen storage disease enterocolitis, and there was a significant correlation between $\mathrm{C}$-reactive protein and hemoglobin levels $(P=0.036)$.

Conclusion: Anemia is a common manifestation of both glycogen storage disease $\mathrm{Ia}$ and $\mathrm{Ib}$, although the pathophysiology appears to be different between these conditions. Those with severe anemia and glycogen storage disease Ia likely have hepatic adenomas, whereas glycogen storage disease enterocolitis should be considered in those with glycogen storage disease Ib.

Genet Med 2012:14(9):795-799

Key Words: anemia; glycogen storage disease I; hepcidin; inflammatory bowel disease; iron
Although anemia has been recognized as a common complication in GSD, there has been limited research on the topic. Prior publications have documented a high incidence of anemia in both GSD Ia and Ib, but there have been few studies examining etiology of this complication. In addition, there are no guidelines for management of this common complication. To have evidence-based recommendations for the associated American College of Medical Genetics consensus guidelines for anemia in patients with GSD I, data from the two largest GSD treatment centers in the United States were obtained. The hematologic data gathered over the past three decades were analyzed in a retrospective manner to describe etiological factors and propose management guidelines for anemia in the GSD I population.

\section{MATERIALS AND METHODS}

Following institutional review board approval at both Duke University and the University of Florida, informed written consent was obtained from all participants, with assent obtained from minor subjects 7 years of age and older who were capable of affirming their desire to participate. Subjects were followed longitudinally at these institutions, and all patients with GSD

\footnotetext{
${ }^{1}$ Glycogen Storage Disease Program and Division of Pediatric Endocrinology, Department of Pediatrics, University of Florida, Gainesville, Florida, USA; ${ }^{2}$ Glycogen Storage Disease Program, Division of Medical Genetics, Duke University Medical Center, Durham, North Carolina, USA; ${ }^{2}$ Department of Statistics, University of Florida, Gainesville, Florida, USA. Correspondence: David A. Weinstein (weinsda@peds.ufl.edu)
} 
I were included in the analysis. Data were available from 202 subjects with GSD I, of whom 163 patients have GSD Ia and 39 patients have GSD Ib. The diagnosis of GSD Ia or Ib was established biochemically or by assessment of enzyme testing from a liver biopsy for all subjects. The diagnosis and subtyping were confirmed in all patients who had mutation analysis (149/163 patients with GSD Ia and 31/39 with GSD Ib).

Patients were evaluated annually with laboratory testing for markers of metabolic control, complete blood counts, inflammatory markers, and ultrasound screening for hepatic and renal lesions. Annual liver imaging with ultrasonography was performed after 5 years of age, and patients discovered to have hepatic adenomas were subsequently monitored more frequently with serial imaging through magnetic resonance imaging, computed tomography scans, or ultrasonography as recommended in the literature. ${ }^{5}$ For patients with GSD Ib, the diagnosis of inflammatory bowel disease was made by pediatric gastroenterologists based on a combination of inflammatory studies, serologic testing, colonoscopies, and capsule endoscopies.

Patient data, including age, gender, current medications, comorbidities, and body mass index, were included in our database. Laboratory values of hemoglobin, hematocrit, mean corpuscular volume, serum iron, total iron-binding capacity, transferrin saturation, ferritin, triglycerides, C-reactive protein, erythrocyte sedimentation rate, uric acid, and lactate were obtained from the most recent encounter. Because many patients with hepatic adenomas underwent surgical resection or liver transplantation and experienced subsequent correction of anemia, all historical laboratory studies were examined to establish the nadir hemoglobin value. Additional concurrent laboratory values obtained at the time of nadir hemoglobin concentration were included for analysis. Anemia status was compared for all patients on the basis of the most recent available laboratory values. Anemia was defined with reference to the National Health and Nutrition Examination Survey (NHANES III), using as the definition hemoglobin concentration that is less than the 5 th percentile of age and gender reference groups. ${ }^{6}$ Severe anemia was defined as hemoglobin $\leq 10 \mathrm{~g} / \mathrm{dl}$. These criteria were defined before analysis.

Comparison of covariates at nadir hemoglobin event was performed across quartiles on the basis of hemoglobin values. For categorical variables, to account for small cell counts, a Fisher's exact test was performed to examine differences in observed counts across the hemoglobin groups. For quantitative variables, an analysis of variance was employed to test for differences in covariate means across the hemoglobin quartiles while controlling for age and gender. Analysis of variance was also used to compare covariate means between patients with and without adenomas on the basis of nadir hemoglobin event. A logistic regression was used to model the probability of adenoma occurrence on the basis of hemoglobin levels from all available records. Because multiple records were collected for each patient across the study period, a mixed-model approach was employed to accommodate repeated measures.
In the GSD Ib group, univariate regression was used to test for the significance of the relationship between hemoglobin values and C-reactive protein levels, erythrocyte sedimentation rate, triglyceride levels, standardized body mass index, and uric acid concentration individually. A $P$ value $<0.05$ was used to define statistical significance.

\section{GSD la}

\section{RESULTS}

Of the 163 patients with GSD Ia, 95 were male. The median age was 15 years (range: $1.5-51.8$ years). A total of 46 patients developed hepatocellular adenomas (31\%) at a median age of 16.7 years. The medication regimens of nine patients included ferrous sulfate, and one patient was treated with erythropoietin at the time of the most recent follow-up. Four patients presented with a history of menorrhagia. Fifteen patients presented with comorbid nephropathy, and two patients had renal failure. One patient had undergone a bone marrow transplant for chronic myelogenous leukemia that was performed 5 years before inclusion in this study.

Overall, 68 of 163 (41.7\%) patients were anemic at their most recent encounter, and more than half of patients over age 15 were anemic (Table 1). Severe anemia was present in 8 of 163 patients (4.9\%), and 6 of $8(75 \%)$ had concomitant hepatic adenomas. Of the 68 anemic patients, 40 patients were examined with complete iron studies. Of patients with iron studies, 32 of 40 had features consistent with iron deficiency anemia with low transferrin saturation and low iron values. Two anemic patients exhibited transferrin saturation values $>40 \%$, normal mean corpuscular volume values, and iron values $>150 \mu \mathrm{g} / \mathrm{dl}$, consistent with anemia of chronic disease. Notably, both of these patients had hepatic adenomas.

Nadir hemoglobin values were compared in quartiles (Table 2). Hepatocellular adenoma was prevalent in $57.9 \%$ of patients in the lowest quartile (hemoglobin $<10.4 \mathrm{~g} / \mathrm{dl}$ ) and was significantly associated with quartile divisions $(P=0.0165)$.

Table 1 Mean hemoglobin values of patients with glycogen storage disease type la and prevalence of anemia and severe anemia at most recent follow-up

\begin{tabular}{lcccc}
\multirow{2}{*}{$\begin{array}{l}\text { Age } \\
\text { category } \\
\text { (years) }\end{array}$} & $\boldsymbol{n}$ & $\begin{array}{c}\text { Hemoglobin } \\
\mathbf{( g / d \mathbf { l } )}\end{array}$ & Anemia & Severe anemia \\
\cline { 3 - 5 } & 3 & $12.0(0.70)$ & $0(0)$ & $0(0)$ \\
\hline $1-2$ & 15 & $12.2(0.93)$ & $1(6.7)$ & $0(0)$ \\
\hline $3-5$ & 19 & $12.3(0.95)$ & $4(21.1)$ & $0(0)$ \\
\hline $6-8$ & 22 & $12.2(0.83)$ & $8(36.4)$ & $0(0)$ \\
\hline $9-11$ & 19 & $12.4(0.79)$ & $3(15.8)$ & $0(0)$ \\
\hline $12-14$ & 19 & $12.8(1.77)$ & $10(52.6)$ & $0(0)$ \\
\hline $15-19$ & 32 & $12.6(1.67)$ & $21(65.6)$ & $1(3.1)$ \\
\hline $20-29$ & 24 & $12.1(2.14)$ & $14(58.3)$ & $3(12.5)$ \\
\hline $30-39$ & 8 & $10.2(2.85)$ & $6(75)$ & $4(50)$ \\
\hline $40-49$ & 2 & $12.5(0.21)$ & $1(50)$ & $0(0)$ \\
\hline $50-59$ & & & & $n(\%)$ \\
\hline
\end{tabular}


Table 2 Nadir hemoglobin quartiles of patients with glycogen storage disease type la

\begin{tabular}{|c|c|c|c|c|c|}
\hline \multirow[b]{2}{*}{ Covariate } & \multicolumn{4}{|c|}{ Hemoglobin } & \multirow[b]{2}{*}{$P$ value } \\
\hline & $\begin{array}{l}\text { Quartile } 1 \\
(2.6-10.3)\end{array}$ & $\begin{array}{c}\text { Quartile } 2 \\
(10.3-11.5)\end{array}$ & $\begin{array}{c}\text { Quartile } 3 \\
(11.5-12.3)\end{array}$ & $\begin{array}{c}\text { Quartile } 4 \\
(12.3-18.5)\end{array}$ & \\
\hline \multirow{2}{*}{ Triglycerides (mg/dl) } & $702.2(474.01)$ & $504.9(426.12)$ & $422.1(280.35)$ & $427.5(269.57)$ & \multirow{2}{*}{0.0113} \\
\hline & $n=17$ & $n=19$ & $n=33$ & $n=32$ & \\
\hline \multirow{2}{*}{ Iron ( $\mu \mathrm{g} / \mathrm{dl})$} & $37.6(15.60)$ & $80.1(39.46)$ & 69.7 (32.09) & $75.2(29.33)$ & \multirow{2}{*}{0.0087} \\
\hline & $n=11$ & $n=9$ & $n=26$ & $n=26$ & \\
\hline \multirow{2}{*}{$\begin{array}{l}\text { Transferrin } \\
\text { saturation (\%) }\end{array}$} & $9.1(3.74)$ & $21.6(10.13)$ & $18.0(7.50)$ & $19.8(7.44)$ & \multirow{2}{*}{0.0046} \\
\hline & $n=10$ & $n=8$ & $n=25$ & $n=26$ & \\
\hline
\end{tabular}

Additionally, serum triglyceride concentration was negatively associated with nadir hemoglobin values $(P=0.0113)$. When a comparison was performed on the basis of adenoma status (Table 3), a relationship was again found between adenomas and anemia. In subjects with adenomas, the mean nadir hemoglobin was $10.3 \mathrm{~g} / \mathrm{dl}(\mathrm{SD}=2.42 \mathrm{~g} / \mathrm{dl})$ as compared with patients without adenomas, who had a nadir of $11.6 \mathrm{~g} / \mathrm{dl}(\mathrm{SD}=1.64 \mathrm{~g} / \mathrm{dl}$; $P=0.0002$ ). Serum triglyceride and serum lactate levels were significantly different between these groups.

Hemoglobin values were compared for all patients who underwent hepatic resection or liver transplantation for hepatocellular adenomas (Table 4). Pre-resection nadir hemoglobin value was compared with hemoglobin level at most recent follow-up. Studies from the operative admission were excluded from comparison.

\section{GSD lb}

Of the 39 patients with GSD Ib , 18 were male. The median age was 13.4 years (range: 1-38 years). Neutropenia was present in all subjects, GSD enterocolitis (inflammatory bowel disease) was diagnosed in 20 patients (51\%), and a total of 6 patients had development of hepatocellular adenomas (15\%). Of the 28 patients with known medication histories, all patients received granulocyte colony-stimulating factor, four patients were on ferrous sulfate supplements and one included erythropoietin. One patient presented with dialysis-dependent renal failure.

In subjects with GSD Ib, 14/21 females (66.7\%) and 14/18 males $(77.8 \%)$ were classified as anemic at most recent follow-up. As a group, 28/39 patients with GSD Ib were anemic (71.8\%). Severe anemia was present in $16 / 39$ patients (41\%). Mean hemoglobin level in patients with GSD Ib was $10.3 \mathrm{~g} / \mathrm{dl}$ $(\mathrm{SD} \pm 1.4)$ in females and $10.2 \mathrm{~g} / \mathrm{dl}(\mathrm{SD} \pm 1.6)$ in males.

An analysis of variance for inflammatory markers and markers of metabolic control demonstrated significant correlation between C-reactive protein and hemoglobin levels $(P=0.036$, Table 5). Correlation between erythrocyte sedimentation rate and hemoglobin levels approached significance $(P=0.071)$, whereas markers of metabolic control did not demonstrate significant correlation.
Table 3 Characteristics of patients with GSD la at time of nadir hemoglobin concentration according to adenoma status

\begin{tabular}{|c|c|c|c|}
\hline & Adenoma & No adenoma & ANOVA \\
\hline Covariate & Mean (SD) & Mean (SD) & $P$ value \\
\hline \multirow[t]{2}{*}{ Hemoglobin (g/dl) } & $10.3(2.42)$ & $11.6(1.64)$ & 0.0002 \\
\hline & $n=55$ & $n=96$ & \\
\hline \multirow[t]{2}{*}{$\mathrm{MCV}(\mathrm{fl})$} & $84.9(9.01)$ & $82.2(5.59)$ & 0.555 \\
\hline & $n=43$ & $n=64$ & \\
\hline \multirow[t]{2}{*}{$\operatorname{Iron}(\mu \mathrm{g} / \mathrm{dl})$} & $89.2(45.54)$ & $64.9(25.94)$ & 0.0112 \\
\hline & $n=14$ & $n=52$ & \\
\hline \multirow[t]{2}{*}{$\mathrm{TIBC}(\mu \mathrm{g} / \mathrm{dl})$} & $423.1(90.40)$ & $393.4(79.50)$ & 0.2459 \\
\hline & $n=13$ & $n=52$ & \\
\hline \multirow[t]{2}{*}{ Transferrin saturation (\%) } & $22.8(11.18)$ & $17.3(6.82)$ & 0.0316 \\
\hline & $n=12$ & $n=51$ & \\
\hline \multirow[t]{2}{*}{ Ferritin (ng/ml) } & $549.2(1146.3)$ & $69.4(54.05)$ & 0.0111 \\
\hline & $n=6$ & $n=36$ & \\
\hline \multirow[t]{2}{*}{ Triglycerides (mg/dl) } & $697.2(436.32)$ & 416.6 (278.99) & 0.0003 \\
\hline & $n=30$ & $n=64$ & \\
\hline \multirow[t]{2}{*}{ Lactate (mmol/l) } & $6.2(9.93)$ & $2.4(1.30)$ & 0.0073 \\
\hline & $n=26$ & $n=53$ & \\
\hline \multirow[t]{2}{*}{ BMI SDS } & $0.6(0.47)$ & $1.8(0.82)$ & 0.0047 \\
\hline & $n=5$ & $n=16$ & \\
\hline
\end{tabular}

ANOVA, analysis of variance; BMI, body mass index; GSD, glycogen storage disease; SDS, standard deviation score; TIBC, total iron-binding capacity.

SI conversion factors: To convert hemoglobin to $\mathrm{g} / \mathrm{L}$, multiply by 10 ; iron to $\mu \mathrm{mol} / \mathrm{L}$, multiply by $0.179 ; \mathrm{TIBC}$ iron to $\mu \mathrm{mol} / \mathrm{L}$, multiply by 0.179 ; ferritin to pmol/L, multiply by 2.247; and triglycerides to $\mathrm{mmol} / \mathrm{L}$, multiply by 0.0113 .

\section{DISCUSSION}

\section{GSD la}

Concordant with previously reported studies of patients with GSD I, we found a high prevalence of anemia in this patient population. When compared with the NHANES reference groups, over half of the patients with GSD Ia who were above the age of 15 years were anemic. Of note, patients were less likely to be anemic in preadolescent age groups, and this may be related to several factors, including lower prevalence of hepatic adenomas, differences in nutrition and metabolic control, cornstarch regimen 
Table 4 Comparison of hemoglobin before and after hepatocellular adenoma resection in patients with GSD la

\begin{tabular}{|c|c|c|c|c|c|c|c|c|c|}
\hline \multirow[b]{2}{*}{ Subject } & \multirow[b]{2}{*}{ Sex } & \multicolumn{4}{|c|}{ Before resection } & \multicolumn{4}{|c|}{ After resection } \\
\hline & & Age & $\begin{array}{l}\mathrm{Hgb} \\
(\mathrm{g} / \mathrm{dl})\end{array}$ & $\begin{array}{l}\text { Hct } \\
(\%)\end{array}$ & $\begin{array}{c}\text { MCV } \\
\text { (cu } \\
\text { micron) }\end{array}$ & Age & $\mathrm{Hgb}$ & Hct & MCV \\
\hline 1 & M & 14 & 11.5 & 33.7 & & 16 & 13.6 & 40.1 & \\
\hline 2 & $\mathrm{~F}$ & 16 & 10.3 & 32 & 83 & 24 & 10.4 & 33 & 91 \\
\hline 3 & M & 17 & 10.3 & 32 & 92 & 28 & 11.7 & 34 & 89 \\
\hline 4 & $\mathrm{~F}$ & 19 & 10 & 30 & 78.3 & 26 & 14.1 & 37.2 & \\
\hline 5 & $M$ & 20 & 10.1 & 30 & 82 & 31 & 16.9 & 48 & 85 \\
\hline 6 & $\mathrm{~F}$ & 21 & 9.8 & 30 & 92 & 36 & 12.9 & 38 & 85 \\
\hline 7 & $M$ & 23 & 14.4 & 42 & 91 & 32 & 16.4 & 46 & 91 \\
\hline 8 & $\mathrm{~F}$ & 30 & 13.6 & 40.3 & & 31 & 13.8 & 38.3 & \\
\hline 9 & $\mathrm{~F}$ & 36 & 9.4 & 30 & 100 & 41 & 12.4 & 39 & 92 \\
\hline 10 & $\mathrm{~F}$ & 45 & 9.8 & 29.8 & & 52 & 12.3 & 38.3 & \\
\hline 11 & $\mathrm{M}$ & 49 & 11.2 & 34 & 84 & 50 & 12.6 & 38 & 91 \\
\hline
\end{tabular}

F, female; GSD, glycogen storage disease; Hct, hematocrit; Hgb, hemoglobin; $\mathrm{M}$, male; MCV, mean corpuscular volume.

Table 5 ANOVA of hemoglobin values in patients with GSD lb

Covariate

ANOVA ( $P$ value)

\begin{tabular}{ll}
\hline $\operatorname{CRP}(n=28)$ & 0.036 \\
$\operatorname{ESR}(n=29)$ & 0.071 \\
$\operatorname{Trig}(n=36)$ & 0.341 \\
BMI SDS $(n=23)$ & 0.414 \\
Uric acid $(n=29)$ & 0.198
\end{tabular}

ANOVA, analysis of variance; BMI, body mass index; CRP, C-reactive protein; ESR, erythrocyte sedimentation rate; GSD, glycogen storage disease; Trig, triglyceride.

adherence, and fewer comorbidities such as menorrhagia and renal failure. The prevalence of anemia in our adult patient population is slightly higher than that seen in the European Study on Glycogen Storage Disease Type I. ${ }^{2}$ It is noteworthy that the standards used to define anemia were different between the two studies. In this study, we controlled for both age and gender, resulting in variable normal ranges; in contrast, a lower limit of $11.9 \mathrm{~g} / \mathrm{dl}$ was used for all patients over age 10 years in the European Study on Glycogen Storage Disease Type I. The prevalence in this study, however, was less than that reported by Talente et al., ${ }^{7}$ who found $81 \%$ of their cohort of 32 patients with anemia.

Patients with GSD may be predisposed to iron deficiency because cornstarch reduces iron bioavailability in the gastrointestinal tract. ${ }^{8}$ Although iron supplementation usually will treat people with mild anemia, patients with severe anemia are often resistant to iron therapy even when administered intravenously. ${ }^{9}$ Patients with more severe anemia were found with a very high adenoma burden. When analyzed, patients with hepatic adenomas had lower hemoglobin values, but serum iron concentrations were greater than average. Taken together, patients with adenomas appear to exhibit anemia of chronic disease that is likely related to increased expression of hepcidin within adenoma tissue. Abnormal regulation of hepcidin in the adenomas results in impaired intestinal absorption of iron and decreased recycling of iron by macrophages. ${ }^{10}$ Notably, most anemic patients with hepatic adenomas demonstrated normalization of hemoglobin levels after adenoma resection or liver transplantation (Table 4).

Several covariates of adenoma status at the nadir hemoglobin event correlated significantly, including serum triglyceride concentration and serum lactate concentration. Although some subjects were included in both studies, this evidence from a larger cohort of subjects and an additional GSD specialty center corroborates previously published data, which demonstrate that metabolic control may play a role in hepatocellular adenoma development. ${ }^{11}$ It is important to note that metabolic control by itself, however, cannot fully explain adenoma formation, and there are genetic and other factors also involved with development of adenomas. ${ }^{12}$ The most strongly correlated laboratory parameter with adenoma formation was serum triglyceride concentration $(P=0.0003)$, which is the most reliable surrogate marker of metabolic control due to stable concentrations over longer periods of time. Serum lactate concentration was also correlated $(P=0.0073)$, although it should be noted that lactate values fluctuate rapidly throughout the day in response to diet and treatment conditions.

Iron supplementation remains the primary therapy for patients with mild anemia. If iron therapy fails or if severe anemia develops, imaging of the liver is warranted if not recently performed to screen for adenoma growth or changing lesions. In the presence of adenomas, intravenous iron therapy may help overcome decreased intestinal iron absorption induced by hepcidin. In the absence of alternative treatment options, severe iron-refractory anemia secondary to hepatocellular adenoma may be best addressed definitively with surgical resection. ${ }^{13}$

\section{GSD lb}

Anemia is more prevalent in patients with GSD Ib (71.8\%) as compared with GSD Ia (41.7\%). Additionally, prevalence of severe anemia is increased in GSD Ib (41\%) as compared with patients with GSD Ia (4.9\%). The observed prevalence of anemia in our GSD Ib population is higher than previously reported findings in the European Study on Glycogen Storage Disease Type I, in which 23 of 56 patients across all age groups were anemic (41\%), but the cohort in this study (median age 13.4 years) was older than in the prior report (median age 7.1 years). ${ }^{2}$

The association between anemia and inflammatory bowel disease in the GSD Ib population is similar to the high prevalence of anemia seen within the non-GSD inflammatory bowel disease population. Burbige et al. ${ }^{14}$ studied 58 ambulatory patients with Crohn disease and reported an anemia prevalence of $51.7 \%$ with hemoglobin values $<11.0 \mathrm{~g} / \mathrm{dl}$. Another large study by Schreiber et al. ${ }^{15}$ found $26.3 \%$ of 334 ambulatory Crohn disease patients with hemoglobin values $<10.0 \mathrm{~g} / \mathrm{dl}$. The etiology of anemia in patients with inflammatory bowel disease is likely attributed to iron deficiency anemia, blood loss, and anemia of chronic 
disease. Blood loss, folate deficiency, inflammation, malnutrition, hemolysis, and marrow-suppressing medications may also contribute to iron deficiency anemia in this population. ${ }^{16}$ Mechanisms for anemia of chronic disease have been hypothesized. It is thought that cytokine release (particularly interleukin-6) from the inflamed intestine stimulates hepcidin synthesis in the liver with resultant anemia of chronic disease. ${ }^{17}$

In our study, the severity of anemia is significantly correlated with the levels of C-reactive protein. The relationship between inflammatory bowel disease severity and anemia in patients with GSD Ib has not been previously documented, and our study is the first to describe this correlation. New-onset development of anemia in a patient with GSD Ib should trigger an evaluation of GSD enterocolitis if a diagnosis has not been made. In contrast to GSD Ia, hepatic adenomas are less common in this population (15\%), and treatment of the inflammatory bowel disease and iron supplementation should result in improvement of anemia.

\section{Conclusion}

Anemia is a common complication in both GSD Ia and Ib, but the cause of this complication appears to be different. Although mild iron deficiency is common in both GSD Ia and Ib due to dietary restrictions, altered iron absorption from cornstarch therapy, and anemia of chronic disease, severe anemia in GSD Ia appears to be related to large hepatic adenoma formation. Likewise, GSD enterocolitis should be considered in GSD Ib patients with severe anemia. Development of severe anemia during puberty or adulthood warrants imaging of the abdomen to search for a bleed or growth of the lesions. In contrast, evaluation for GSD-associated inflammatory bowel disease should occur in patients with GSD Ib who develop severe anemia. Improved understanding of the pathogenesis of this complication should allow new therapies to be introduced to treat anemia in the future.

\section{ACKNOWLEDGMENTS}

Support for this project was provided in part by the National Institutes of Health and National Center for Research Resources CTSA grants 1UL1RR029890 (University of Florida) and 5UL1RR024128-03 (Duke University). Additional philanthropic support was provided from the Scott Miller Glycogen Storage Disease Program Fund, the Type Ib Glycogen Storage Disease Research Fund, and the Efforts 4 Ellie Fund.

\section{DISCLOSURE}

The authors declare no conflict of interest.

\section{REFERENCES}

1. Kishnani PS, Koeberl D, Chen YT. Glycogen storage diseases. In: Valle D, Beaudet AL, Vogelstein B, et al. (eds). The Online Metabolic \& Molecular Bases of Inherited Disease, ch. 71. McGraw-Hill: New York, 2008. (http:// www.ommbid.com).

2. Rake JP, Visser G, Labrune P, Leonard JV, Ullrich K, Smit GP. Glycogen storage disease type I: diagnosis, management, clinical course and outcome. Results of the European Study on Glycogen Storage Disease Type I (ESGSD I). Eur J Pediatr 2002;161 Suppl 1:S20-S34.

3. Weinstein DA, Wolfsdorf JI. Effect of continuous glucose therapy with uncooked cornstarch on the long-term clinical course of type 1a glycogen storage disease. Eur J Pediatr 2002;161 Suppl 1:S35-S39.

4. Visser G, Rake JP, Labrune P, et al.; European Study on Glycogen Storage Disease Type I. Consensus guidelines for management of glycogen storage disease type 1b - European Study on Glycogen Storage Disease Type 1. Eur J Pediatr 2002;161 Suppl 1:S120-S123.

5. Franco LM, Krishnamurthy V, Bali D, et al. Hepatocellular carcinoma in glycogen storage disease type la: a case series. J Inherit Metab Dis 2005;28:153-162.

6. National Center for Health Statistics. Plan and operation of the third National Health and Nutrition Examination Survey, 1988-94. Vital Health Stat 1994;1:32.

7. Talente GM, Coleman RA, Alter C, et al. Glycogen storage disease in adults. Ann Intern Med 1994;120:218-226.

8. Amine EK, Hegsted DM. Effect of diet on iron absorption in iron-deficient rats. J Nutr 1971;101:927-935.

9. Weinstein DA, Roy CN, Fleming MD, Loda MF, Wolfsdorf Jl, Andrews NC. Inappropriate expression of hepcidin is associated with siron refractory anemia: implications for the anemia of chronic disease. Blood 2002;100:3776-3781.

10. Roy CN, Weinstein DA, Andrews NC. 2002 E. Mead Johnson Award for Research in Pediatrics Lecture: the molecular biology of the anemia of chronic disease: a hypothesis. Pediatr Res 2003;53:507-512.

11. Wang DQ, Fiske LM, Carreras CT, Weinstein DA. Natural history of hepatocellular adenoma formation in glycogen storage disease type I. J Pediatr 2011;159:442-446.

12. Kishnani PS, Chuang TP, Bali D, et al. Chromosomal and genetic alterations in human hepatocellular adenomas associated with type la glycogen storage disease. Hum Mol Genet 2009;18:4781-4790.

13. Reddy SK, Kishnani PS, Sullivan JA, et al. Resection of hepatocellular adenoma in patients with glycogen storage disease type la. J Hepatol 2007;47:658-663.

14. Burbige EJ, Huang SH, Bayless TM. Clinical manifestations of Crohn's disease in children and adolescents. Pediatrics 1975;55:866-871.

15. Schreiber S, Howaldt S, Schnoor M, et al. Recombinant erythropoietin for the treatment of anemia in inflammatory bowel disease. N Engl J Med 1996;334:619-623.

16. Wilson A, Reyes E, Ofman J. Prevalence and outcomes of anemia in inflammatory bowel disease: a systemic review of the literature. Am J Med 2004;116 Suppl 7A:44S-49S.

17. Semrin G, Fishman DS, Bousvaros A, et al. Impaired intestinal iron absorption in Crohn's disease correlates with disease activity and markers of inflammation. Inflamm Bowel Dis 2006;12:1101-1106. 